\title{
About complex refractive index of black Si
}

\author{
Emil Pinčík ${ }^{*}$, Robert Brunner*, Hikaru Kobayashi ${ }^{* *}$, Milan Mikula**
}

\begin{abstract}
The paper deals with the complex refractive index in the IR light region of two types of samples (i) as prepared black silicon, and (ii) thermally oxidized black silicon (BSi) nano-crystalline specimens produced both by the surface structure chemical transfer method using catalytic Ag evaporated spots (as prepared sample) and by the catalytic Pt catalytic mesh (thermally oxidized sample). We present, compare, and discuss the values of the IR complex refractive index obtained by calculation using the Kramers-Krönig transformation. Results indicate that small differences between optical properties of as prepared black Si and thermally oxidized BSi are given by: (i) - oxidation procedure, (ii) - thickness of the formed black Si layer, mainly, not by utilization of different catalytic metals, and by iii) the different thickness. Contamination of the surface by different catalytic metals contributes almost equally to the calculated values of the corresponding complex refractive index.
\end{abstract}

K e y w o r d s: black silicon, porous silicon, complex refractive index, catalytic mesh

\section{Introduction}

Black silicon structures are used in the development of the new type of Si-based solar cells without anti-reflection coatings. Their own anti-reflection properties enable the formation of solar cells with conversion efficiencies over $19 \%$ on large areas. The conversion efficiency of corresponding solar cell can be increased by suitable passivation of defects created during etching procedure. The oxidation of prepared black silicon structures is one of the most suitable passivation procedures. Si nano-crystallites covered by $\mathrm{SiO}_{x}$ create one of the basic elements of $\mathrm{BSi}$ structures used for the development of corresponding high efficiency solar cells.

The SSCT - is a surface structure chemical transfer method, can produce a nano-crystalline Si black color layer on $\mathrm{c}$-Si with a range of thickness of $\approx 50 \mathrm{~nm}$ to $\approx 300 \mathrm{~nm}$ by the contact of $\mathrm{c}-\mathrm{Si}$ immersed in chemical solutions $\mathrm{HF}+\mathrm{H}_{2} \mathrm{O}_{2}$ with a catalytic mesh.

The second type of porous Si structures were formed by standard electrochemical manner in solution of $\mathrm{HF}$ and methanol under influence of the electric field between the Si sample (+) and Pt electrode (-).

Researchers at ISIR Osaka University developed a fabrication method of low reflectivity $\mathrm{Si}$ surfaces in which a mold with catalytic $\mathrm{Pt}$ layer is contacted with $\mathrm{Si}$ immersed $\mathrm{H}_{2} \mathrm{O}_{2}+\mathrm{HF}$ solution [6]. The PL maximum of a black silicon layer prepared on polycrystalline $\mathrm{Si}$ wafer at room temperature had the position $\approx 1.85 \mathrm{eV},[1]$. A similar method was also applied for the preparation of the BSi samples in this contribution. An additional alter- native technological approach was developed in the same Japanese laboratories by M. Takahashi et al [2].

In the contribution [3] we presented an analysis of the photoluminescence properties of as prepared thin $\approx 100$ $\mathrm{nm}$ thick) multicolor silicon structures.

In this paper we will present and discuss values of the calculated complex refractive index of two types of black silicon structures differing by high temperature after growth thermal treatment and by used catalytic surface metal $\mathrm{Pt}$ or $\mathrm{Ag}$.

\section{Experiment}

\subsection{Preparation of samples}

Two types of samples were prepared. P-type (100) Si has been treated using (i) $\mathrm{Pt}$ mash and (ii) $\mathrm{Ag}$ spots in the solution $15 \mathrm{wt} \% \mathrm{HF}+25 \mathrm{wt} \% \mathrm{H}_{2} \mathrm{O}_{2}$. The first one, produced in the ISIR Osaka University, Japan, with Pt mash has been after the BSi growth few minutes oxidized at $900{ }^{\circ} \mathrm{C}$. The second one, prepared in the Institute of Physics SAS Bratislava, Slovakia, with very thin Ag evaporated spots was not annealed, only dried. The estimated thickness of the sample with $\mathrm{Pt}$ is $\approx 200 \mathrm{~nm}$. The estimated thickness of the second one with $\mathrm{Ag}$ is $\approx$ $100 \mathrm{~nm}$.

\subsection{Theory}

For calculation of complex refractive index of structures we used IR reflectivity measurements recorded by a Digilab Excalibur FTS 3000 MX spectrometer with

\footnotetext{
* Institute of Physics, SAS, Dúbravská cesta 9, 84511 Bratislava, Slovakia, emil.pincik@savba.sk, ** Institute of Scientific and Industrial Research, Osaka University, Mihogaoka, Ibaraki, Osaka 567-0047, Japan, *** Faculty of Chemical and Food Technology of STU, Radlinského 9, 81237 Bratislava, Slovakia
} 
the FTIR spectroscopy software: ResolutionsPro 5.2.0, Agilent with Kramers-Krönig transformation. A PIKE Technologies Specular Reflectance Accessory was used at an angle of 30. The diameter of the measured area was $10 \mathrm{~mm}$, used resolution: $4 \mathrm{~cm}^{-1}$, number of scans: 60 .

Real and imaginary parts of complex refractive index can be expressed from following equations

$$
\begin{aligned}
& n=\frac{1-r^{2}}{1+r^{2}-2 r \cos \theta} \\
& k=\frac{-2 r \sin \theta}{1+r^{2}-2 r \cos \theta}
\end{aligned}
$$

Where $n$ and $k$ are real and imaginary parts of refractive index $\hat{n}=n-i k, R=r^{2}$ is experimentally measured reflectance, and $\theta$ is the phase difference between incident and reflected waves. It can be calculated as, [4]

$$
\theta_{c}=2 \frac{\omega_{c}}{\pi} \int_{0}^{\infty} \frac{\ln r(\omega)-\ln r\left(\omega_{c}\right)}{\omega^{2}-\omega_{c}^{2}} \mathrm{~d} \omega
$$

where $\theta_{c}$ is the quantity $\theta$ at given frequency $\omega_{c}$.

\subsection{Experimental results and Discussion}

The contribution is dealing with the formation of porous black silicon structures prepared electrochemically in different solutions. Usually nanocrystalline black silicon is formed by electrochemical reaction (solution $\mathrm{HF}+$ $\mathrm{H}_{2} \mathrm{O}_{2}$ ) utilizing catalytic mesh (SSCT) and evaporated very thin Ag spots. The used ions of the chemical solution penetrate under the influence of the internal electrical field through the space region of the nanocrystalline Si layer, and consequently, the nanocrystalline Si layer grows.

Figure 1 illustrates IR dispersion characteristics of reflectance of black silicon structures prepared with Pt (BSi ref 1) catalytic mesh and Ag (samp.5) catalytic spots.

Figure 2 shows IR dispersion characteristics of refractive index of black silicon structures prepared with $\mathrm{Pt}$ (BSi ref) catalytic mesh and Ag (samp. 5) catalytic spots. In the region $500 \mathrm{~cm}^{-1} 1200 \mathrm{~cm}^{-1}$ is, in the both records, increased the value of the refractive index due to increased absorbance of the investigated surface due to Si-O and Si$\mathrm{OH}$ absorbance bands see also Fig.1.

Figure 3 illustrates IR dispersion characteristics of extinction coefficient $k$ of black silicon prepared with the $\mathrm{Pt}$ (BSi ref) catalytic mesh and Ag (samp.5) catalytic spots.

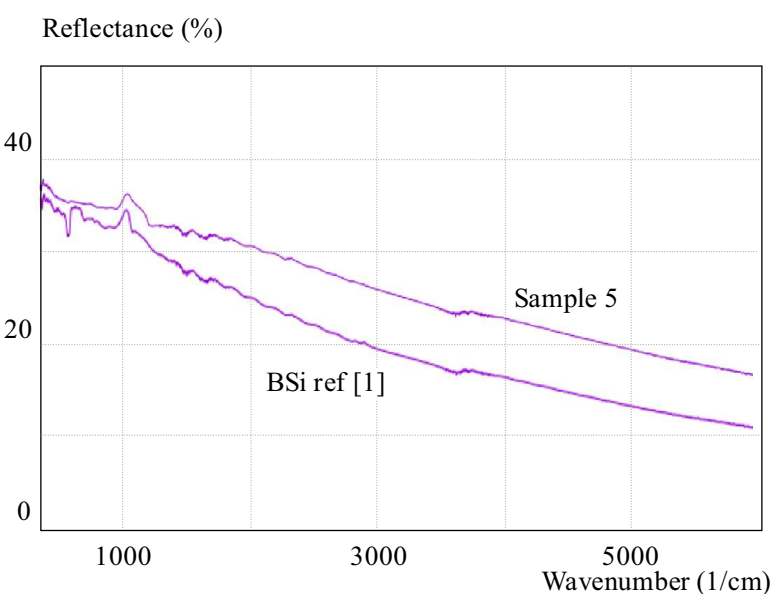

Fig. 1. IR dispersion characteristics of reflectance of black silicon structures prepared with $\mathrm{Pt}$ (BSi ref 1) catalytic mesh and $\mathrm{Ag}$ (samp.5) catalytic spots

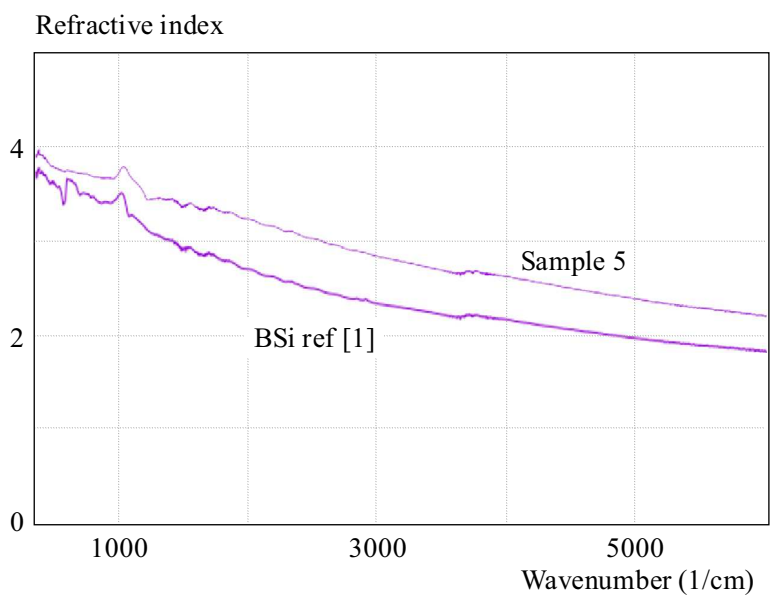

Fig. 2. IR dispersion characteristics of refractive index of black silicon structures prepared with PT ( BSi ref ) catalytic mesh and $\mathrm{Ag}$ (samp.5) catalytic spots

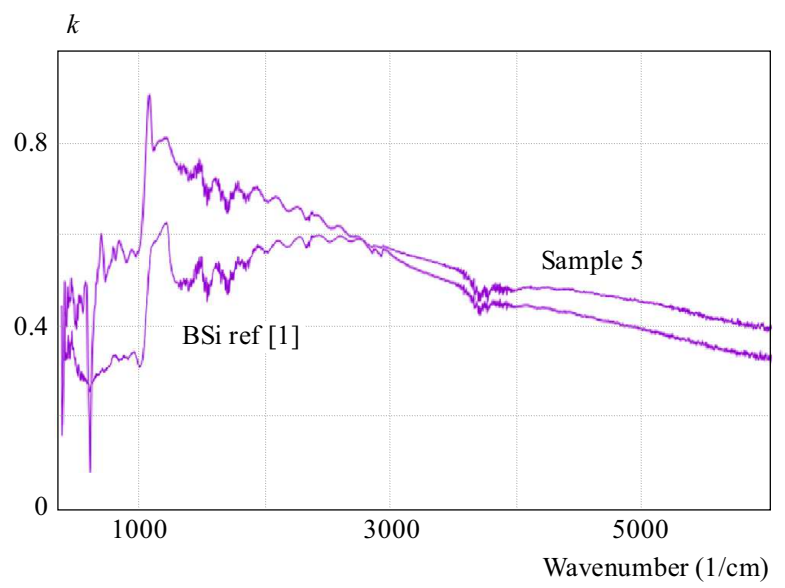

Fig. 3. IR dispersion characteristics of extinction coefficient $k$ of black silicon prepared with the PT (BSi ref) catalytic mesh and Ag (samp.5) catalytic spots.

Results indicate that small differences between optical properties of as prepared black Si and thermally oxidized $\mathrm{BSi}$ is given by (i) oxidation procedure, (ii) thickness of 
the formed black Si layer, mainly, not by utilization of different catalytic metals, and by the different thickness of the black silicon layer. Contamination of the surface by different catalytic metals contributes almost equally to the calculated values of the corresponding complex refractive index.

\section{Conclusion}

The contribution deals with the complex refractive index in the IR light region of two types of samples (i) as prepared black silicon and (ii) thermally oxidized black silicon (BSi) nano-crystalline specimens produced both by the surface structure chemical transfer method using catalytic Ag evaporated spots (as prepared sample) and by the catalytic Pt catalytic mesh (thermally oxidized sample). We present, compare, and discuss mainly the values of the IR complex refractive index obtained by calculation using the Kramers-Kronig transformation. Results indicate that small differences between optical properties of as prepared black Si and thermally oxidized BSi is given by (i) oxidation procedure, (ii) thickness of the formed black Si layer, mainly, not by utilization of different catalytic metals, and by the different thickness of the black silicon layer. Contamination of the surface by different catalytic metals contributes almost equally to the calculated values of the corresponding complex refractive index.

\section{Acknowledgements}

Following agencies: APVV project No.0152/15 and VEGA projects Nos.2/0076/15, 1/0900/16 partly financially supported this contribution. We would like to thank Prof. K. Imamura of ISIR Osaka University, Japan, Mrs. S.Bacova, and Mr. Peter Zitto (both of the IoP SAS Bratislava) for the preparation of samples and very insightful discussions. We thank for the help with this contribution to Ms. Julia Tomekova of the FMPI Comenius University, Bratislava, Slovakia, also.

\section{REFERENCES}

[1] K. Imamura, F. C. Franco, Jr. T. Matsumoto, and H. Kobayashi, "Ultra-low reflectivity polycrystalline silicon surfaces formed by surface structure chemical transfer method", Appl. Phys. Lett., 103 (1) 2013 013110, doi:10.1063/1.4813089.

[2] M. Takahashi, T. Fukushima, Y. Seino, W. -B. Kim, K. Imamura and H. Kobayashi, "Surface Structure Chemical Transfer Method for Formation of Ultralow Reflectivity Si Surfaces", J. Electrochem. Soc., 160(8) (2013) H443, doi:10.1149/2.044308jes.

[3] E. Pincik, R. Brunner, H. Kobayashi, M. Mikula, P. Vojtek, J. Greguš, Z. Zabudla, K. Imamura and P. Švec Jr., "The photoluminescence of multicolor silicon", Journal of the Chinese Advanced Materials Society, vol. 4, no. 2, pp. 152-171, http://dx.doi.org/10.1080/22243682.2016.1151374.

[4] D. Palik Edward (Ed.), Handbook of Optical Constants of Solids, Academic Press Inc., Orlando, 1985.

Received 23 April 2017 